\title{
Techniques and Results of Observations of Rapid and Ultrarapid Variable Stars
}

\author{
BRIAN WARNER (Austin)
}

\begin{abstract}
Introduction
The inception of the present era of interest in rapidly varying stars may be identified with M.F. WALKER's discovery in 1954 (WALKER 1954) of changes in the brightness of the blue object MacRae $+43^{\circ} 1$ (now known as MVLyr), on a time scale of a few minutes. Outside of flare stars, few other stellar objects were suspected to vary on such a short time scale. GREENSTEIN's (1954) spectra showed that MVLyr resembles an old nova, which stimulated WALKER to look for light fluctuations in old, recurrent, and dwarf novae and related stars. His observational evidence that all members of these classes show variations of various amplitudes and time scales is summarized in the IAU Colloquitm on non-stable stars (WALKER 1957). Most spectacular of his discoveries was the existence of $71 \mathrm{sec}$ periodic variations in the light from the remnant of Nova Herculis 1934 (DQ Her) (WALKER 1956).

Since these pioneering investigations a great deal of observation time has been devoted to elucidating the general nature of the stellar systems showing such flickering. Most information has been derived from the rather slower changes associated with the general binary nature of these variations, though even here the short periods (generally $4-6$ hours, but others down to the 82 minute period of WZ Sge) make good photometric and spectroscopic time resolution, especially during eclipses, essential. These studies, pursued primarily by KRAFT, HERBIG, WALKER, MUMFORD, SMAK, KRZEMINSKI and associates, used conventional photoelectric photometry and spectroscopy, with the exception of the recent valuable introduction of the electronographic camera (WALKER 1966, WALKER and CHINCARINI 1968). With the advent of modern high speed electronic techniques we are now beginning to take a fresh interest in the rapid flickering, the discovery of which was the initial stimulus to this whole field of investigation.
\end{abstract}

The interpretation of the general light curves resulting from the binary character of the cataclysmic variables will be discussed in a later section of this colloquium and have been previously reviewed by KRAFT (1963). Here I will review briefly the modern methods available for study of rapid changes of brightness in stars, and some of the results obtained from applications of these techniques.

\section{Techniques}

Nearly all the objects of interest are fainter than magnitude 12 , and the majority are fainter than magnitude 14 . If we are to study rapid light variations in such objects it goes without saying that large telescope aperture and high overall quantum efficiency are objectives to be aimed at. By comparison with conventional UBV photometry some requirements may be relaxed, but others need additional attention.

The general light curves for objects such as cataclysmic variables at minimum light can be obtained with time resolutions of the order of minutes with conventional analogue (d. c.) photometry. Much of what is known of these objects has been obtained in this manner using telescopes of about 36-inch aperture. For studies in which the principal aim is to investigate the detailed variations with time (either of the flickering or of eclipses) we may be compelled to use as broad band as possible (i. e., "white light" - no filters interposed) and to monitor continuously. The generally high flux in the blue and ultraviolet region of rapid variables, coupled with relatively high quantum efficiency of blue-sensitive photomultipliers, is to our advantage here.

Because of the emission line spectra of most of our objects, it is no hardship to abandon the UBV system with its required $1 \mathrm{P} 21$ phototube. There are many photomultipliers now available with alkali cathodes having quantum efficiencies in the blue in excess of 20 percent; 
examples are RCA 8575, EMI 9634QR, EMI 9750 and AMPEREX 56DVP, all with 2-inch cathodes, and RCA 4516 with $3 / 4$-inch cathode. In our experience the Amperex tube has the lowest dark noise (NATHER and WARNER 1971), and can be used uncooled for most broad band high speed photometric applications. Recent improvements in red and infrared sensitivity should make possible at least one minute time resolution at $\sim 16$ magnitude at longer wavelengths.

With the 82-inch Struve reflector, freshly aluminized, we obtain typically 10,000 counts per second at $V=15$ for blue variables (in white light). The sky, through a 15 arc-second diameter diaphragm gives $\sim 1200 \mathrm{c} / \mathrm{s}$. Thus we are restricted to 1 percent statistical uncertainty at $1 \mathrm{sec}$ time resolution at $\mathrm{V}=15$ (see later for effects of atmospheric scintillation), but can obtain a signal-to-background of better than unity even at $V=19$ by using a 7 arcsecond diaphragm.

The need for continuous monitoring in order not to lose information on "time structure" brings its own problems. From the above figures, small variations in sky background are unimportant for $\mathrm{V}<16$. Also, we can often tolerate some slow drift in photomultiplier sensitivity, or in atmospheric extinction, because we rarely are interested in intrinsic stellar variations $\sim$ omo1 with time scales of hours. Thus it is usually permissible to abandon observations of standard stars (except for a single observation at the beginning of a run), to make only infrequent sky brightness observations, and to apply mean extinction coefficients.

On the other hand, we obviously require an offset guider, accurate guiding, and either constant atmospheric transparency or continuous monitoring of the transparency. At McDonald Observatory we have met all these requirements simultaneously by constructing a special two channel photometer with automatic guider. The first channel monitors the variable, and the second channel (which contains the auto-guider) monitors a nearby reference star. Sky measurements in both channels are made every 20 minutes or so by moving the telescope. The auto-guider, controlled by the computer described below, can use as little as one percent of the time of the reference channel to determine the position of the reference star and to remove errors by moving the telescope a calculated amount.

Both channels contain 4-position filter wheels driven by stepping motors, so that sequential filter photometry may be carried out. This is a compromise: instead of constructing a multichannel photometer for observing in several colours simultaneously, we simulate such a system by time-sharing on one channel. Filter changing takes $\sim 0.2 \mathrm{sec}$ (it could be made much faster) and different dwell times in each colour are prearranged.

With data arriving at the rate of typically one integration per second in each of the two channels it is evident that the observations should be made as automatic as possible. The simultaneously cheapest, most efficient, and most versatile way of achieving this is to employ a modern small digital computer as the control centre of the observational equipment.

Fig. 1 demonstrates the overall plan of the equipment we use at $\mathrm{McD}$ onald Observatory. It can be readily moved from one telescope to another. The computer (a NOVA 1200, manufactured by Data General Corporation) is programmed to simultaneously read the integrated counts from each channel. There are separate $50 \mathrm{MHz}$ counters in each channel. Integration times can be changed at will from the operator console. The NOVA also sends pulses to the drivers of the stepping motors that change filters. At specified intervals, the computer moves a stepping motor in channel 2 that drives a triangular obscuration across the photometer diaphragm. From the times of appearance and disappearance of the reference star the computer is able to ascertain the position of the star inside the diaphragm and send the appropriate number of pulses to the telescope digital control system to recentre it (and hence also the variable on channel 1). Such an autoguider system can be constructed very cheaply when one already has a digital computer. Sky measurements may be obtained by instructions given through the operator console to the NOVA requesting an offset of a known amount and then returning.

Initializing of the parameters of the program (start time, integration and dwell times, etc.) is made either through the operator console or through the teletype. 


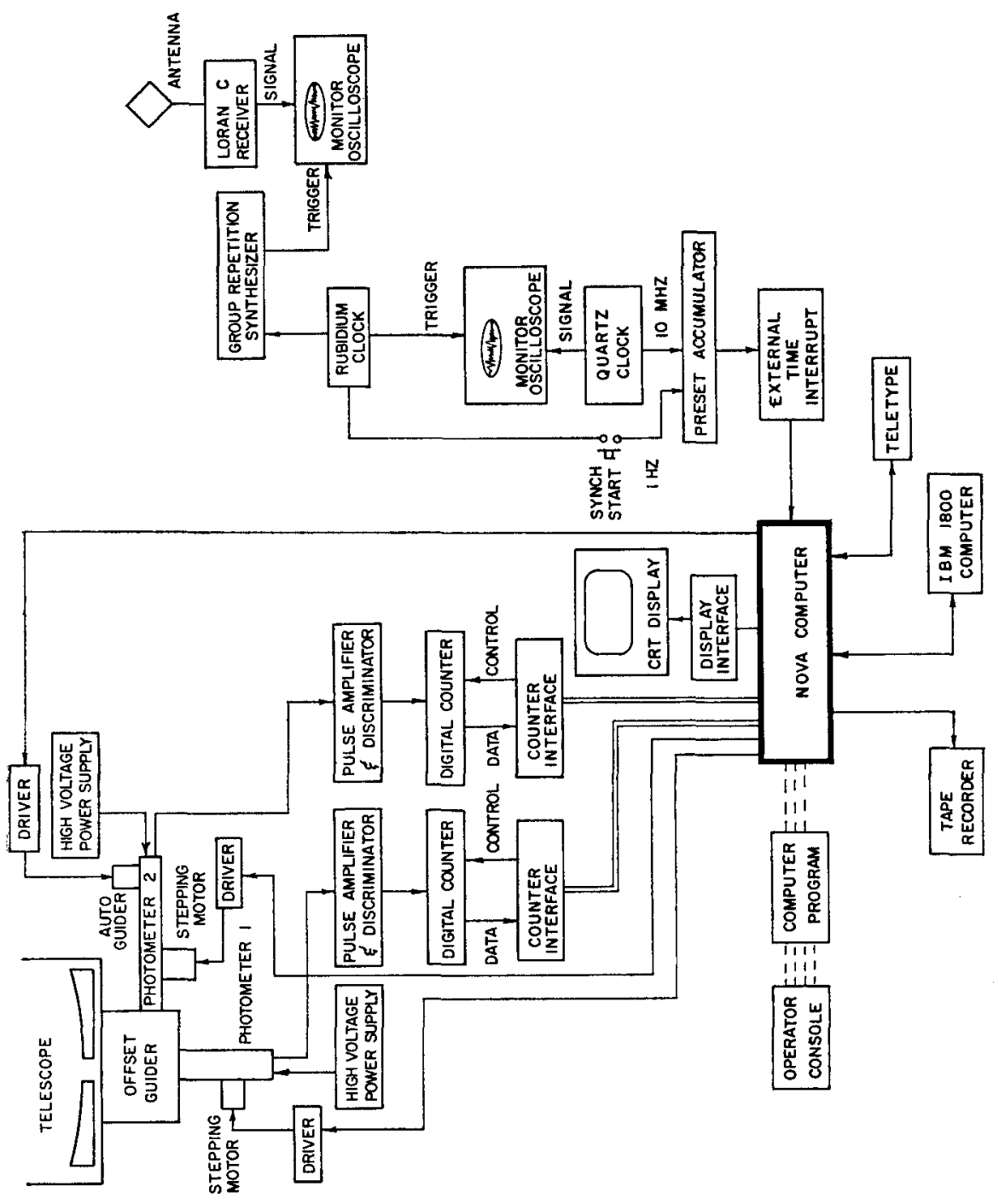

Fig. 1: Schematic diagram of two-channel high speed photometer.

The computer is run by external interrupts from the time system which will be described in the section on pulsar observations. By using a sense switch (on the operator console) as an "enable" switch, the NOVA time counting can be started in phase with the external clock, and thereafter merely counts the external time pulses.

With data arriving at high speed, it is essential to be able to see what is happening. We can no longer afford to wait until the next day (or next month) to evaluate the quality of the night or of the data. We have consequently constructed a sophisticated program that enables the NOVA to display, on an ordinary oscilloscope, the various channels of data that are activated. Thus we are able to inspect in real time a plot of data from each channel, and to select pieces for magnification. Looking at the reference channel tells us if the night is of 
good quality; looking at the data from the variable indicates if our integration time is too long or too short, if the star is particularly active, etc. This makes for very efficient use of telescope time as we are able to perform on-line decisions to make the data more meaningful.

It is also possible to perform some on-line analysis, either in the NOVA or with the IBM 1800, with which the NOVA has a real time link. We will shortly be able to request the NOVA to display a power spectrum (or periodigram analysis) of the data being taken, which it will do by a request to the 1800 for help.

At the rate of a magnitude per second in each channel, we acquire $\sim 4 \times 10^{4}$ magnitude measurements around each typical cataclysmic variable binary period ( 5 hours), $\sim 10^{5}$ magnitudes per night and $\sim 10^{\circ}$ per successful observing run. There are several possibilities open to us for recording this data. Until recently we have been a major supporter of the paper tape manufacturers, but the headaches of transfer to cards or magnetic tape have caused us to obtain a magnetic tape unit for direct recording of all data. This unit is compatible with the CDC 6600 computer on campus in Austin, so most reductions are carried out at the end of the observing run. We tried writing data on line onto the dises of the 1800 , but as this computer is used on line by other telescopes at McDonald Observatory, we could not rely on getting its attention at all times. Reduced data are currently stored on cards and are displayed by computer-controlled graph plotter. We are also able to display the data on the control oscilloscope of the CDC 6600 and manipulate them into suitable form for microfilming or enlarging. (The diagrams in WARNER and NATHER (1971) were made in this fashion.)

If we observe a periodically varying signal with superimposed noise it is well known that the noise level may be reduced by synchronous averaging. This type of technique has been used for upwards of a hundred years by observers of eclipsing binaries to produce average light curves.

We may distinguish two types of noise - that produced by intrinsic light changes in the star, and that caused by atmospheric scintillation and photon counting (i. e. local noise). In the presence of a strong signal, it is clearly best to record "continuously" in order to receive that maximum amount of information. Again this is what is done under conventional observations of eclipsing binaries. However, objects are now known in which the period of light variability is so short that it is advantageous to perform signal averaging in real time. The most obvious example is the optical pulsar in the Crab Nebula. In this case the signal is so small (typically $\sim 100$ detected photons per period) that photon counting noise dominates and the only method of observation is synchronous summing.

Of course, to use real time synchronous signal averaging one must know the period in advance. In the case of the optical pulsar this was initially available from radio observations. Since the first observations of the Crab pulsar (COCKE, DISNEY and TAYLOR 1969; NATHER, WARNER and MacFARLANE 1969) which used relatively crude timing and synchronizing equipment, the data acquisition methods have become considerably more sophisticated. The active study of this object has led to detailed knowledge of both the first and second derivatives of the period, so that on any night one is able to predict the period with considerable accuracy. For the most accurate work it is however still necessary to spend one night determining the best basic period for use on subsequent nights.

The equipment that has been constructed at McDonald Observatory by R. E. NATHER uses the NOVA computer both for applying the technique of synchronous averaging, and also for providing a continuous updating of the period. The latter is performed by precalculating a period ephemeris tape, allowing for pulsar slowdown, earth rotation and earth orbital velocity changes, and this is read by the NOVA. In order to prevent any slip in phase of greater than $1 \mu \mathrm{sec}$ during a night between the instrumental period and the apparent pulsar period update takes place in 100 picosecond steps roughly every 15 seconds.

To make use of this potential timing accuracy it is necessary to employ a clock that does not vary by more than $1 \mu \mathrm{sec}$ per night. We currently use a local $10 \mathrm{MHz}$ crystal oscillator near the NOVA, which is monitored against an atomic clock (see Fig. 1). This atomic clock is frequently checked against the LORAN $C$ transmissions, which enables us to maintain absolute timing accuracy of about $0.5 \mu \mathrm{sec}$. 
In this way we have carried out observations of the Crab Pulsar with 10, 20, 50 and $100 \mu \mathrm{sec}$ time resolution, maintaining our instrumental period so that no phase slippage of greater than a few $\mu$ sec occurred during a night's observation. Typical integration times were 1 minute (1800 pulsar cycles). At the end of an integration, the accumulated light curve is transferred to another part of the computer memory and is read out during the next integration. In this way we can obtain contiguous integrations. This data, when reduced, will be used for a search for short time scale fluctuations in pulse arrival times - i.e. "jitter" in the slow down rate of the pulsar.

\section{1) 2-channel operation}

\section{Observational Results}

First we will discuss some practical aspects of using the two-channel photometer. As we have already indicated, the primary use of our second channel is to obtain information about variations in extinction (i. e. atmospheric transparency). Even on the best of nights there may be slow ( $\sim 1 / 2$-hour timescale) variations in extinction due to movement of large, low density, dust masses (this is particularly true in the Southwest of the U.S.A. during the current drought conditions). The effects of these are readily removed with our second channel. The stringent requirements of "perfect" nights when using a single channel system are largely removed with the use of a second channel. Figure 2 provides an example of this. In this observation, simultaneous 2-second integrations were given on two non-variable stars, on a night with drifting cirro-stratus. Rapid variations of $\sim 1$ magnitude were recorded in both channels. The normalized ratio of the output of the two channels (each with sky removed) is shown in the lower part of Figure 2. It can be seen that no systematic variations appear that can be correlated with variations in the signals. A histogram of the departures of the calculated ratio from the mean is given in Figure 3. Although slightly skew, which is merely a result of the small data sample used, it can be seen that the width of the distribution at $e^{-1}$ height is $\sim 0.023$ magnitudes, giving a noise in each of the two channels of $\sim 1.1$ percent. It may be noted that nowhere in the two channels is the signal stationary enough to make an estimate of the (scintillation) noise level, but 1 percent is typical of a normal photometric night.

We have thus demonstrated that it is possible to carry out high-speed photometry even through (relatively thin) clouds. An immediate advantage is that this increases (by a factor of about two at a normal observing site) the number of nights available for such observations. A further advantage is that reliable high speed photometry becomes possible at large zenith distances. This not only allows one to follow a star for longer runs during a night, but also allows northern hemisphere observers to poach on southern territory. Thus I have a good run on CP Puppis (declination $-35^{\circ}, \mathrm{V} \sim 17$ ) from McDonald Observatory (latitude $+30^{\circ}$ ).

Because the signal-ratio contains the noise of both channels, one has of course to tolerate a somewhat higher noise level ( $\sim$ factor 1.5) than usual, but this is within the range of the usual night-to-night variations in scintillation noise. It should be noted that SERKOWSKI (1971) has shown that absorption by clouds is essentially neutral, so that we do not have to pick a comparison star of the same colour as the variable. It may further be remarked that the above approach breaks down on moonlit cloudy nights - thin clouds appear to be very much better scatterers than absorbers, and the first indication of a cloud during moonlit nights is a large increase in total brightness due to increased sky brightness, followed by a diminution as the starlight becomes absorbed in thicker clouds. The signal-ratio method does not correctly allow for this because we do not make rapid measures of sky brightness changes.

Figure 4 demonstrates what our display system shows on a good photometric night. Here $Z$ Cam is varying on a time scale of $\sim 3$ minutes with an amplitude $\sim$ om15, and there are more rapid variations of $\sim 0 \mathrm{0mo1}-\mathrm{Om} \cdot 2$ on time scales $\sim 10$ seconds. The absence of any similar variations in the reference channel demonstrates the reality of these $Z$ Cam variations. On such a photometric night there is clearly no need to use the signal-ratio method. Indeed, on most nights of variable extinction we can smooth the data in channel 2 , using a time 


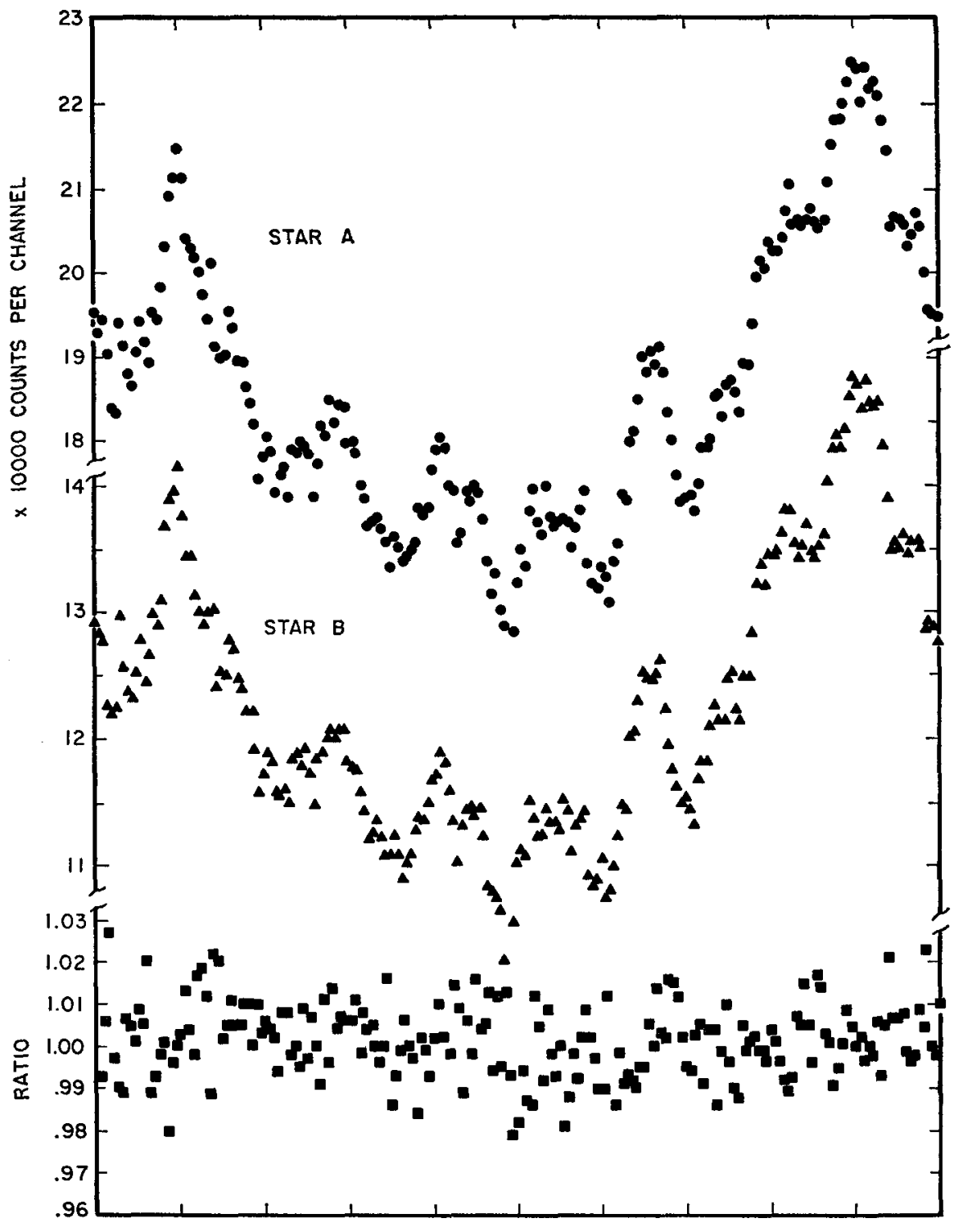

INTEGRATION TIME 2 SECS PER CHANNEL

Fig. 2: Example of use of two-channel photometer on non-variable stars under cloudy conditions.

constant appropriate to the conditions, thereby eliminating most of the noise contribution of the reference channel.

In summary, the second monitor channel is a reliable indicator of perfect photometric nights, allows for the removal of slow variations of transparency without significant increase in noise, and permits removal of rapid changes in transparency with a tolerable increase in noise. 


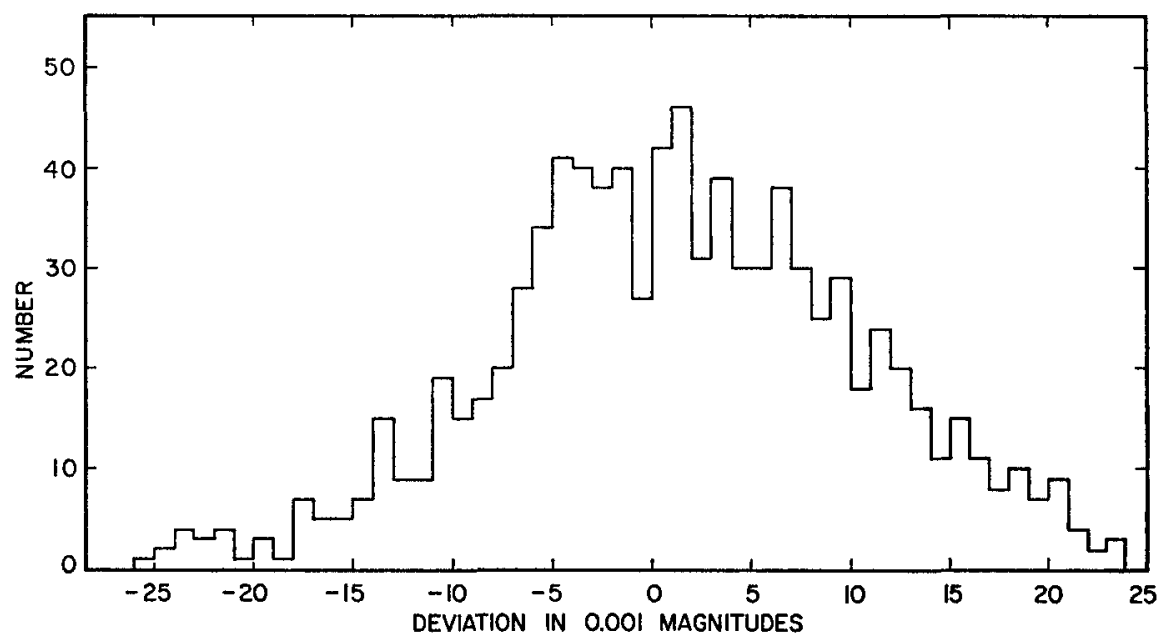

Fig. 3 : Signal-ratio histogram derived from Figure 2.

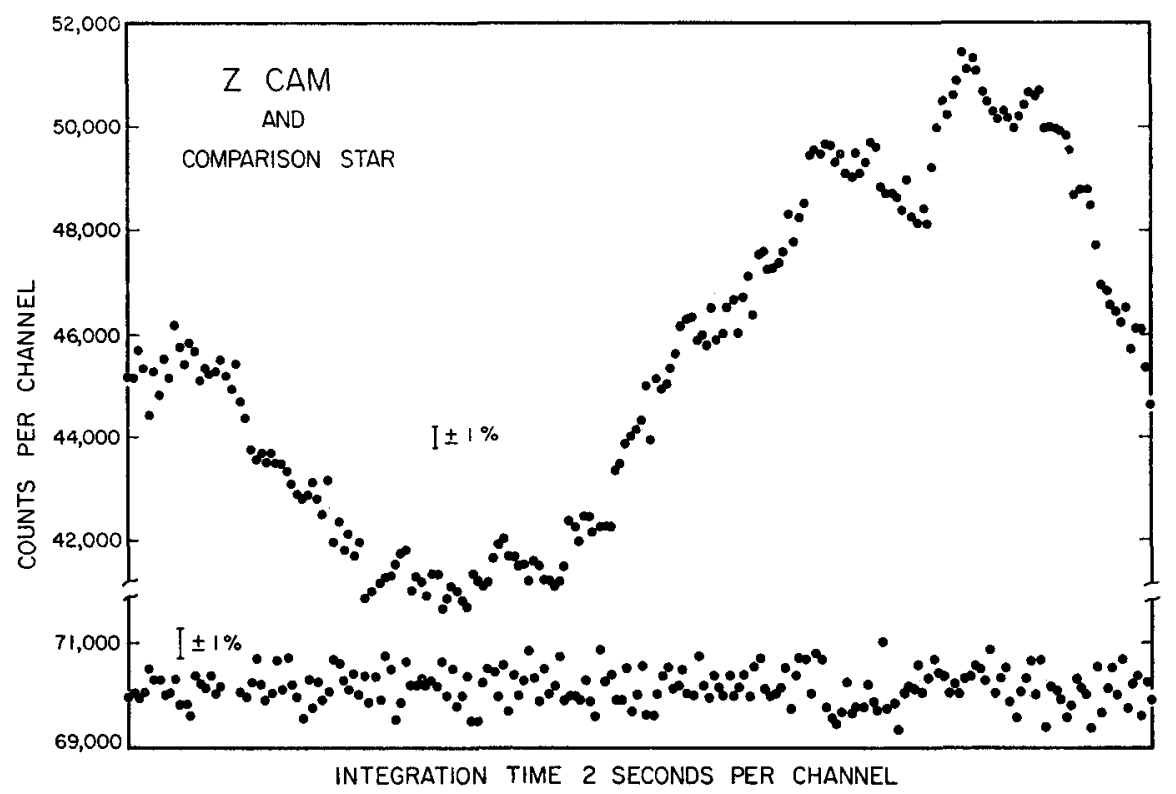

Fig. 4: Z Cam and reference star. 


\section{2) Scintillation}

From Figure 4 it is seen that the limiting factor in observing individual rapid changes in brightness in a star is atmospheric scintillation noise. The peak-to-peak noise in the reference star in Figure 4 is $\sim 2^{1 / 2}$ percent, whereas the noise due to photon counting is $( \pm 2 \sigma) \sim 400 / \sqrt{70,000}=1.5$ percent.

Thus scintillation contributes a band of noise $\sim \pm 1$ percent wide. This is quite typical for 2 second integrations. Because scintillation noise does not fall rapidly with decreasing frequency, there is unusually significant scintillation power even in $10 \mathrm{sec}$ integrations. Occasionally nights are found where the scintillation is only \pm 0.25 percent, and exceptionally it may be as large as \pm 5 percent. In the writer's experience there is only a moderate correlation with size of seeing disc. The one yery bad night ( \pm 5 percent band on all stars even with 5 second integration) coincided with extensive dust.

From the figures given earlier, it is seen that with our equipment, photon counting noise dominates only for $\mathrm{V}>16$.

\section{3) Application to Cataclysmic Variables}

Our most extensive applications of the high speed photometer have been to study the flickering in cataclysmic variables. In order to interpret the light curves illustrated below, some understanding of the model for cataclysmic variables is required. Briefly, we have a close double star, the primary being a white dwarf and the secondary a late-type dwarf losing mass at the inner Lagrangian point. The mass transferred between components forms a ring around the white dwarf. From the presence of a hump in the light curve lasting for half of the orbital period we can deduce that there is a bright spot somewhere in the system. The location of this bright spot has been suggested as either on the surface of the white dwarf or at the edge of the ring. The origin of the flickering has been a mystery, but has been suggested as occurring either in the ring as a whole or on the white dwarf.

Figure 5 illustrates an eclipse of U Gem. Other examples are given by WARNER and NATHER (1971). Before eclipse, at the peak of the hump, there is rapid flickering of large amplitude. Previous studies (KRZEMINSKI 1965, MUMFORD 1963, PACZINSKI 1965) had found variations on a time scale of minutes. We see that the flickering extends down to time scales of seconds. However, during eclipse this flickering disappears.

As the circumstellar ring is only partially eclipsed, we see that we can rule out the ring itself as a contributor to the flickering. The flickering amplitude reaches a maximum at the peak of the hump, which indicates that the flickering is coming from the bright spot. From the fact that the hump lasts for almost exactly half a period, we can deduce that the bright spot has very little extension in longitude. In the case of U Gem, KRZEMINSKI's (1965) model shows that if the bright spot were situated on the white dwarf, it should be eclipsed in only a few seconds. However, Figure 5 shows that $\sim 100$ seconds is required for eclipse of the bright spot (see WARNER and NATHER 1971 for details). We conclude that the bright spot is on the outer edge of the circumstellar ring.

This revised geometry then leads to an entirely new model for U Gem, in which the white dwarf itself is not eclipsed at all. The observed eclipse is due to a total eclipse of the bright spot and a partial eclipse of the ring. A similar model has been independently propesed by SMAK (1971).

We have extensive observations of many cataclysmic variables, but I will give only a few examples.

In Figures 6 and 7 we find that the rapid flickering observed in RW Tri and UX UMa also disappears during eclipse, showing that the same type of model as for U Gem is applicable. In the case of UX UMa, the bright spot is slightly fainter than the white dwarf, so we can see first the white dwarf and then the bright spot emerge from eclipse. The flickering doesn't appear until the bright spot starts to emerge. 


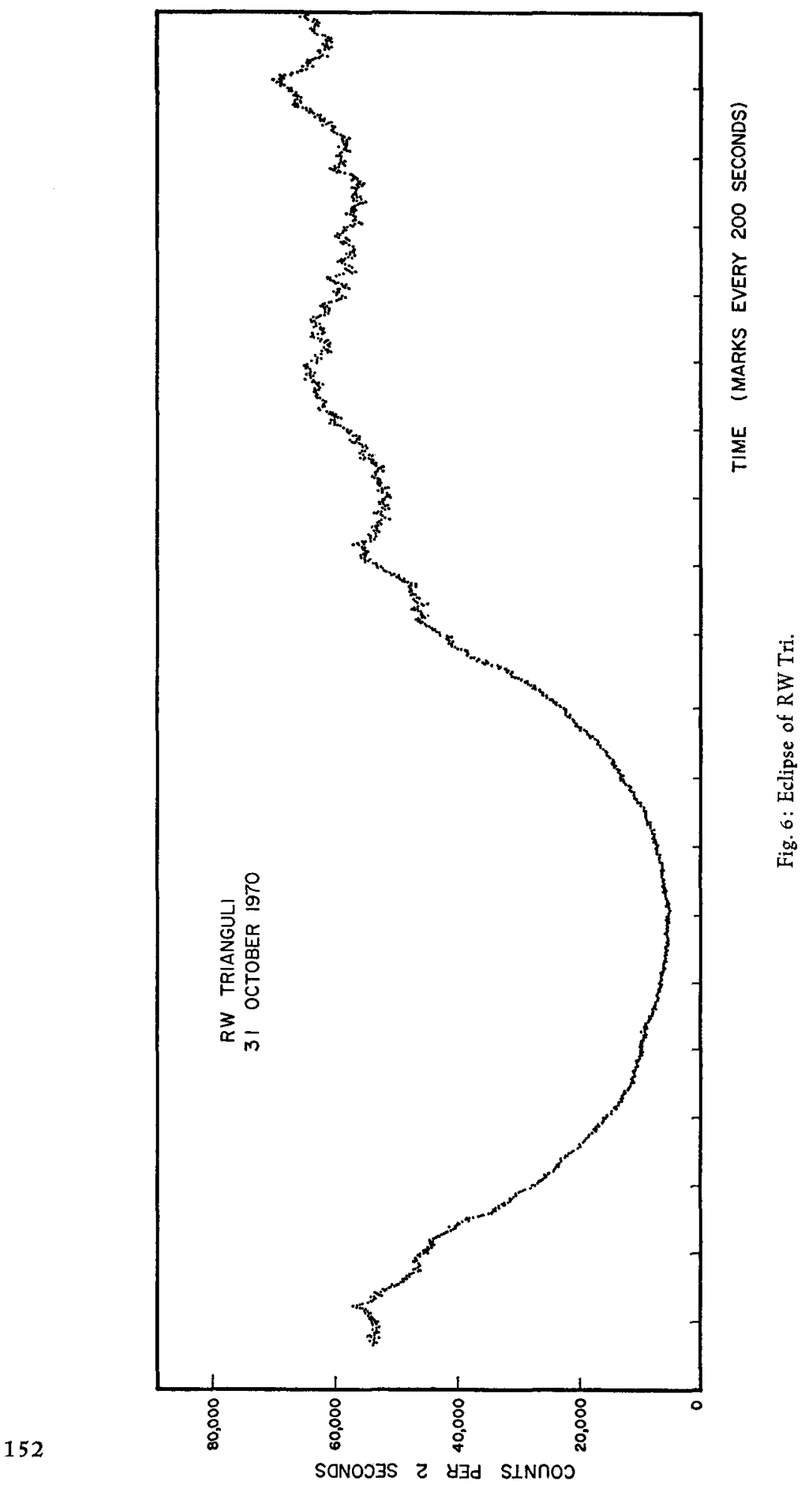




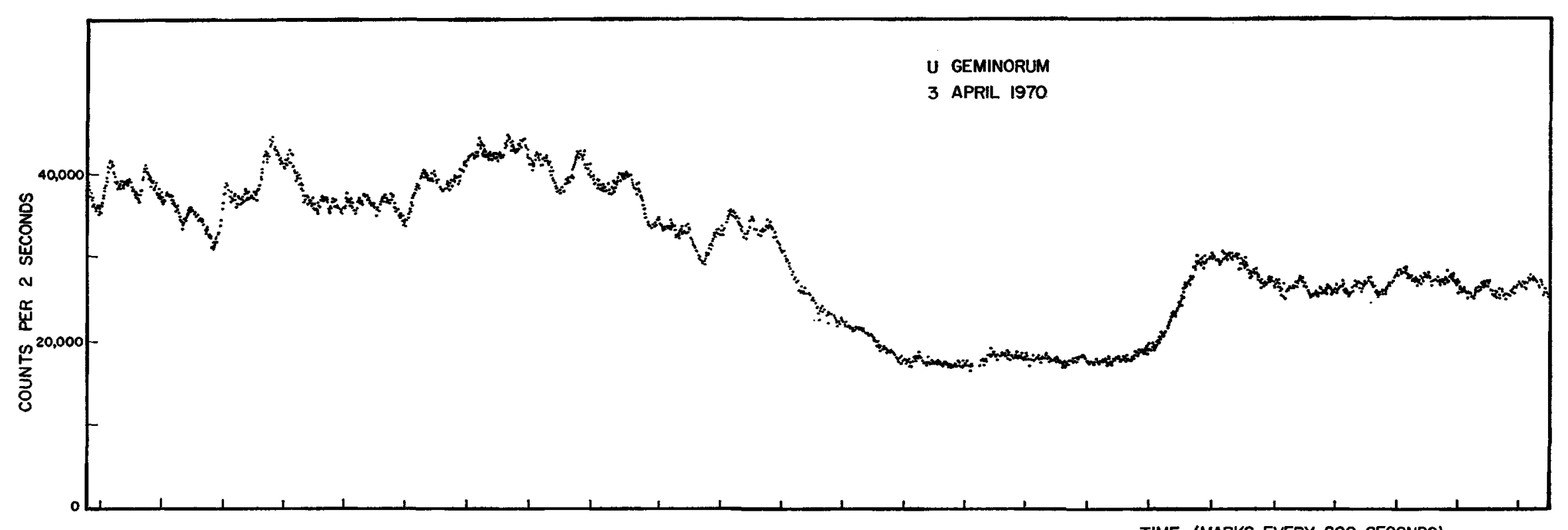

Fig. 5 : Eclipse of U Gem.

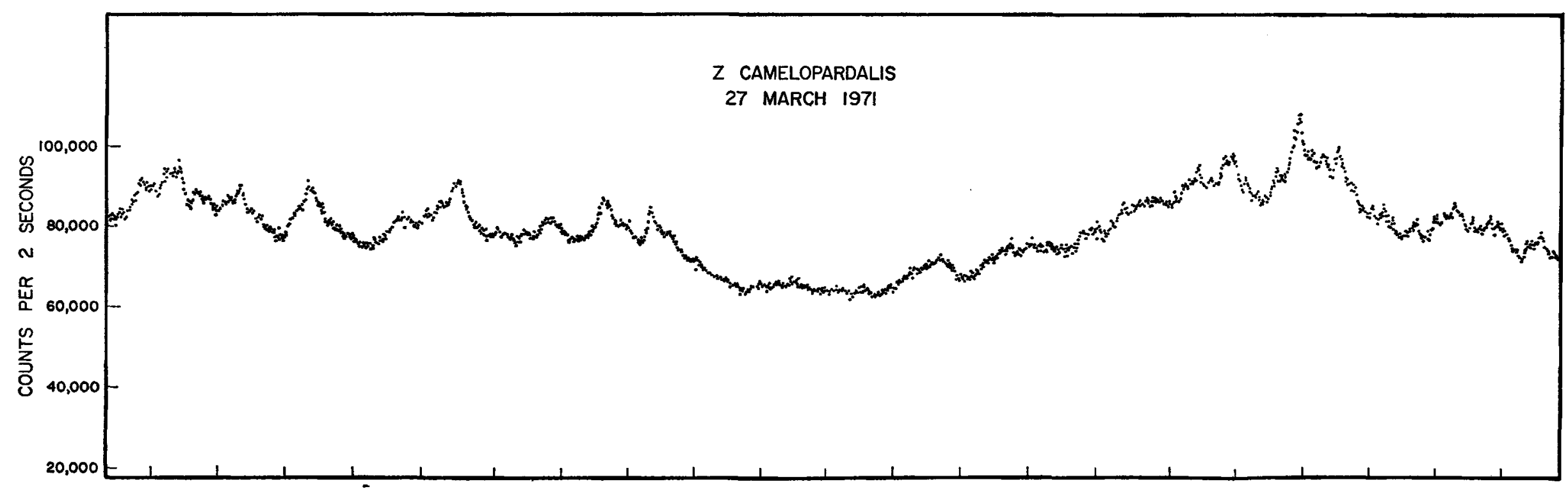




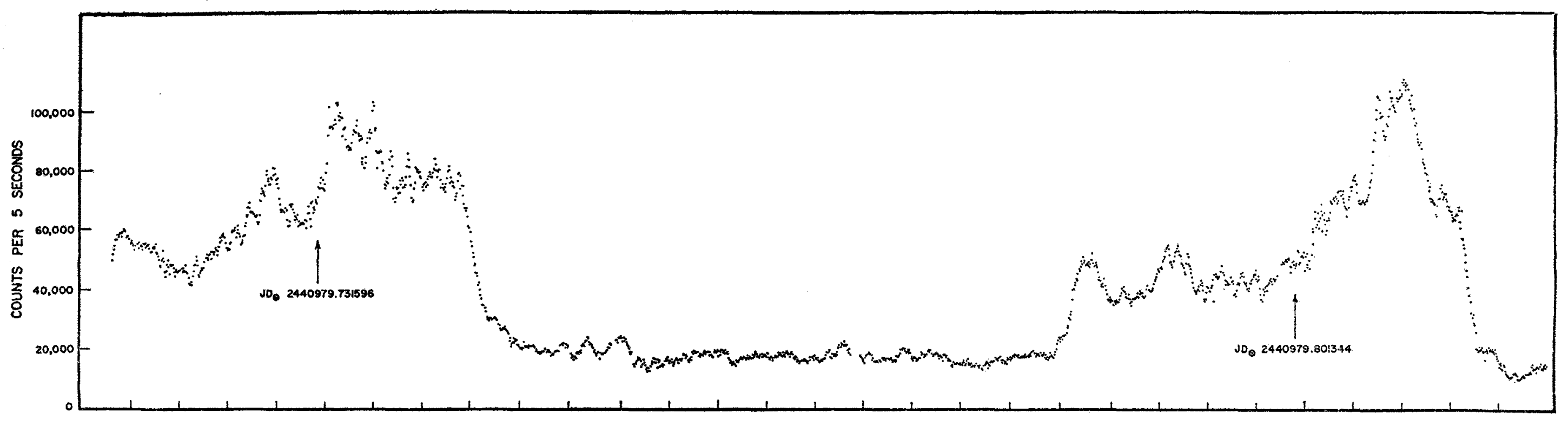

TIME (MARKS EVERY 300 SECONDS)

Fig. 9: Light-curve of VV Pup. Arrows indicate predicted maxima.

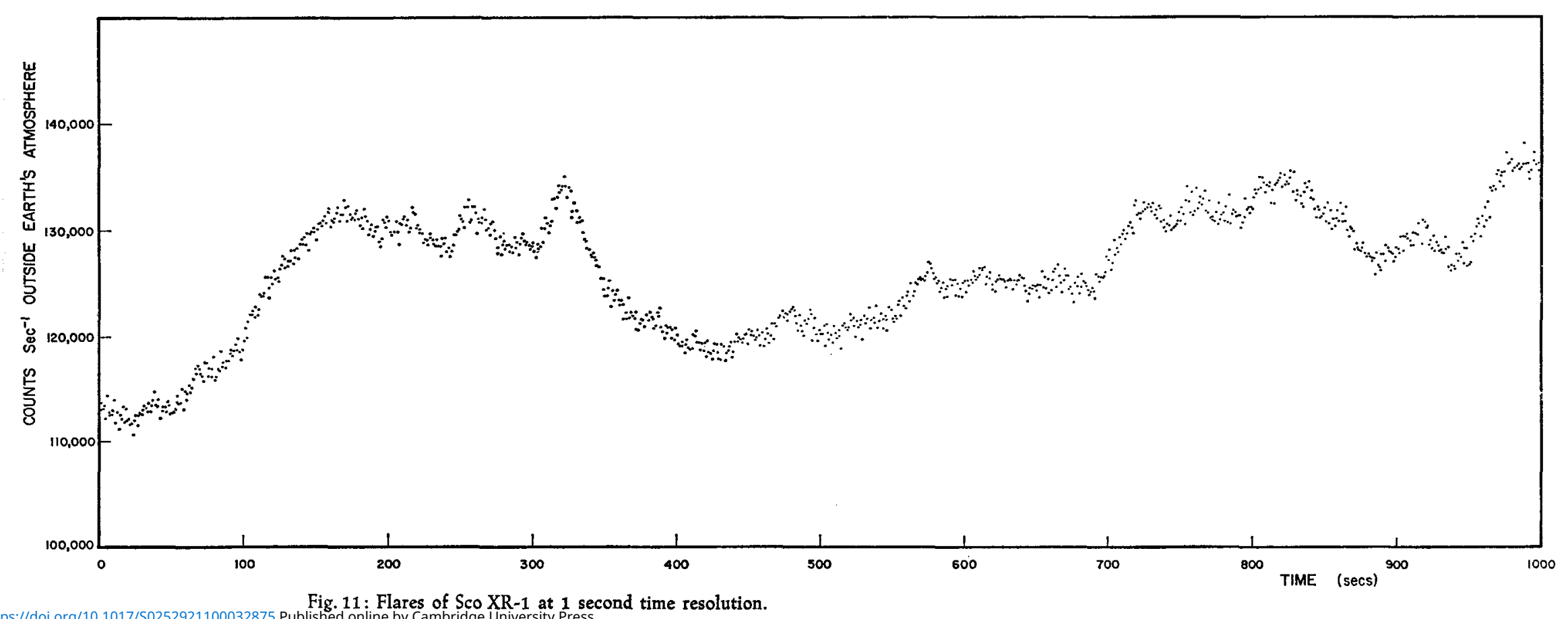




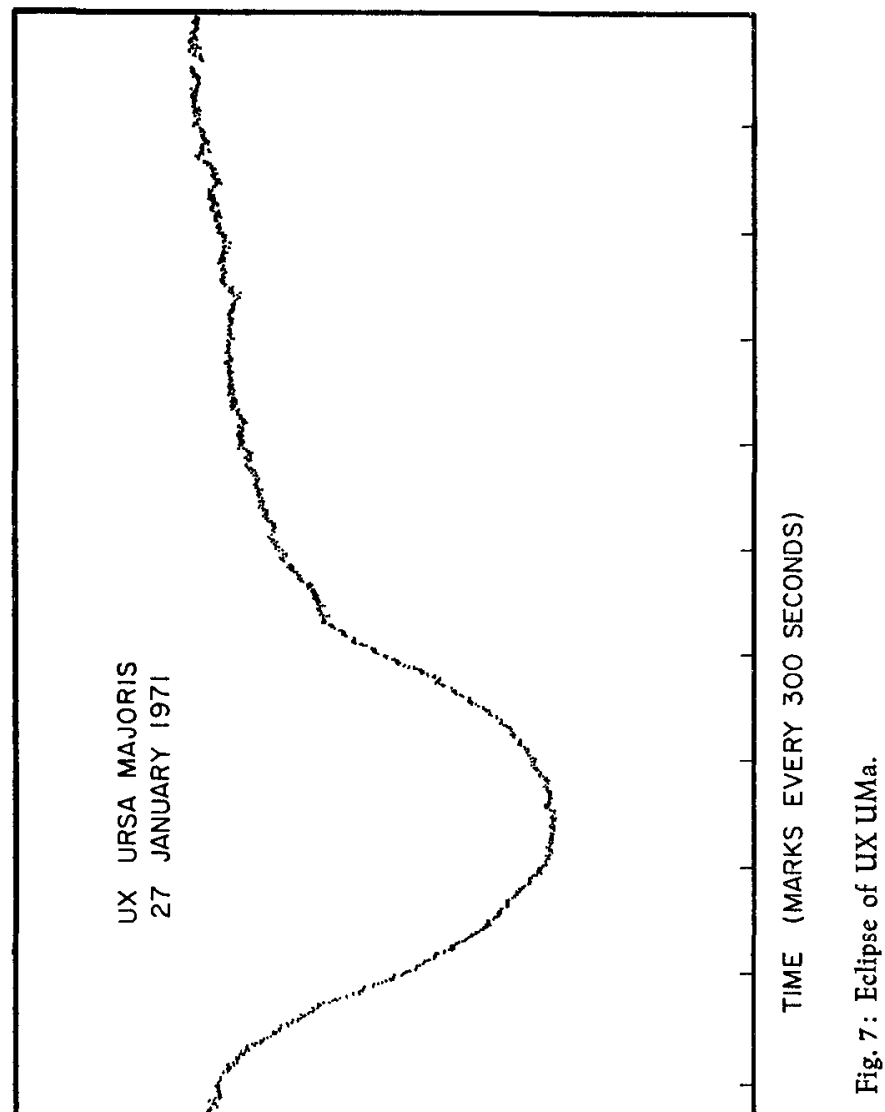


The disappearance of high frequency flickering during eclipse thus appears to be a common feature of cataclysmic variables. This is one of the principle discoveries found from application of high speed photometry. It suggests that we may be able to detect eclipses in other cataclysmic variables in which the eclipse has not been found with conventional photometry. An example is given in Figure 8. No eclipses have been found in Z Cam (KRAFT et al. 1969). However, in Figure 8 we see a region in which the high frequency flickering is depressed for about 20 minutes. This occurs shortly after spectroscopic conjunction - at the same phase that eclipses in other cataclysmic variables occur. The depth of eclipse is no deeper than many other dips in the $Z$ Cam light curve, but the absence of high frequency flickering characterizes an eclipse.

Finally in Figure 9 we show a light curve for the 100 minute binary VV Pup. The presence of very rapid flickering, with maximum amplitude at the peak of the hump is again evident.

\section{4) HL Tau-76}

This white dwarf was discovered to be variable by LANDOLT (1968), and subsequently found to resemble dwarf novae in light curve, but with an amplitude $\sim 0.3$ magnitude and recurrence cycle $\sim 12.5$ minutes (WARNER and NATHER 1970). We have carried out further photometry, and find evidence for a strictly periodic driving mechanism to the light variations of this star, with period $12.437 \pm 0.003$ minutes. Only the strong pulses have this period, and there is some superimposed phase jitter (a light curve was illustrated at the Colloquium but is too large to be reproduced here; it will shortly appear in the Monthly Notices of the Royal Astronomical Society).

Three colour photometry of this star is shown in Figure 10. It can be seen that the star is much bluer at the maximum of a pulse. The change in effective temperature is $\sim 650^{\circ} \mathrm{K}$ and this reasonably accounts for the increase in brightness, showing that no increase in radius occurs during outbursts.

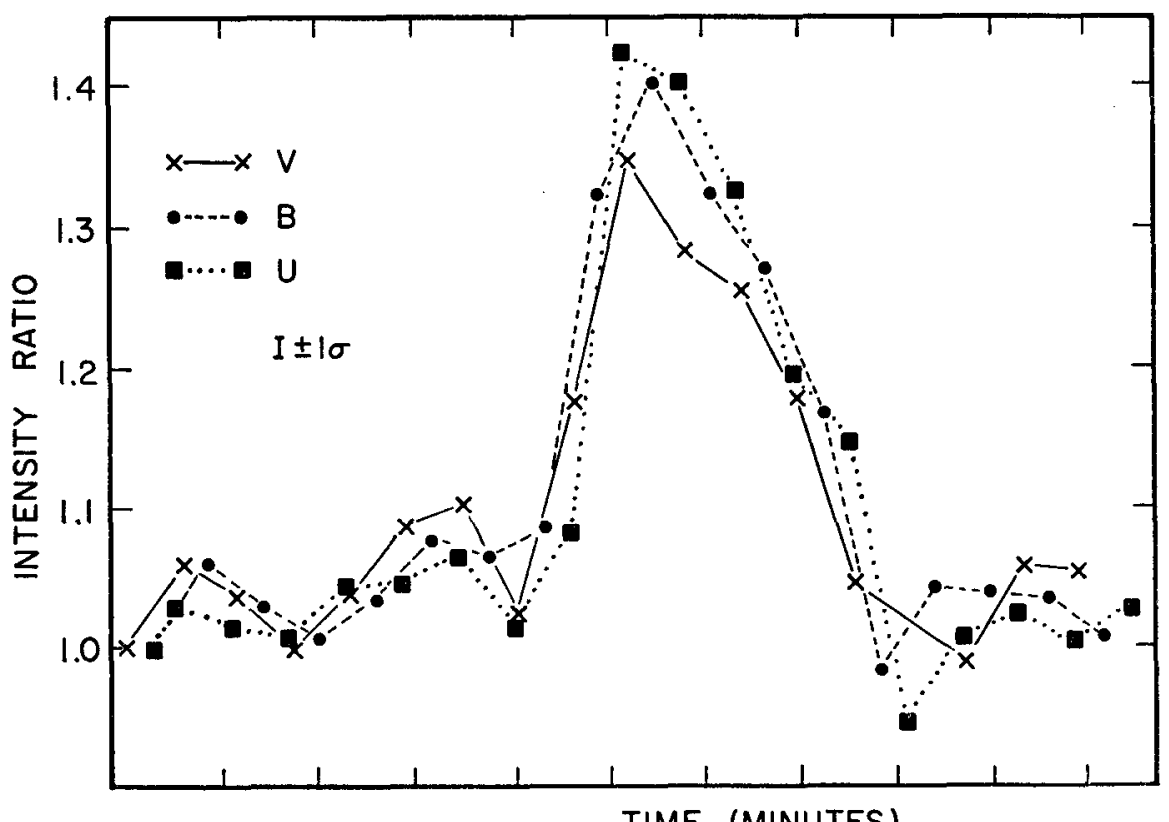

Fig. 10: UBV observations of an outburst of HL Tau-76. 
As ED KELLOGG is giving a paper later in this Colloquium on optical and X-rax emission from cosmic X-ray sources I will confine my remarks to some unpublished work on Sco XR-1.

We have monitored Sco XR-1 on several nights, both during quiet periods and during flares. When Sco XR-1 is flaring we detect typically 70,000 photons $\sec ^{-1}$ in white light on the 82-inch, which is about twice what WESTPHAL et al. (1968) were able to achieve with the 100-inch. Figure 11 illustrates a small piece of a run on the night of March 24, 1971 (U.T.) when Sco XR-1 was particularly active. The resolution is $1 \mathrm{sec}$ and the data has been corrected for atmospheric extinction. The Figure starts at $\mathrm{JD}_{\odot} 2441034.93899$.

Several attempts have been made to find periodic oscillations in the optical light curve of 5 co XR-1, the most intensive being that by LAMPTON et al. (1970). They were unable to find any periodicities. In Figure 12 we show a power spectrum of 6000 secs of the data taken on March 24, 1971. To produce this smoothed spectrum, the data were devided into four portions, with 50 percent overlap in each, linear trends were removed, and the resulting four power spectra averaged. A 10 percent cosine bell data window was added to the data. The power spectrum has not been illustrated beyond $0.10 \mathrm{sec}^{-1}$, it is flat (the mean level is indicated) to $1 \mathrm{sec}^{-1}$, but with no significant features in the noise. It can be seen that Sco XR-1 contributes power above the noise only for frequencies below about $0.06 \mathrm{sec}^{-1}$. At higher frequencies atmospheric scintillation dominates. None of the peaks in the power spectrum is significant; none of them appeared strongly on all of the four individual spectra before averaging. Thus we concur with the conclusion of LAMPTON et al.

We have made one run on Cyg XR-2, on the night of 18 June 1971 (U.T.). This object showed no activity whatever on this occasion.

\section{6) Synchronous Photometry of DQ Herculis}

The 71 second periodic light variations of the white dwarf in the DQHer system, which are rendered difficult to observe because of the flickering activity in the bright spot, are an obvious area where synchronous signal averaging can help to define a light curve. The study

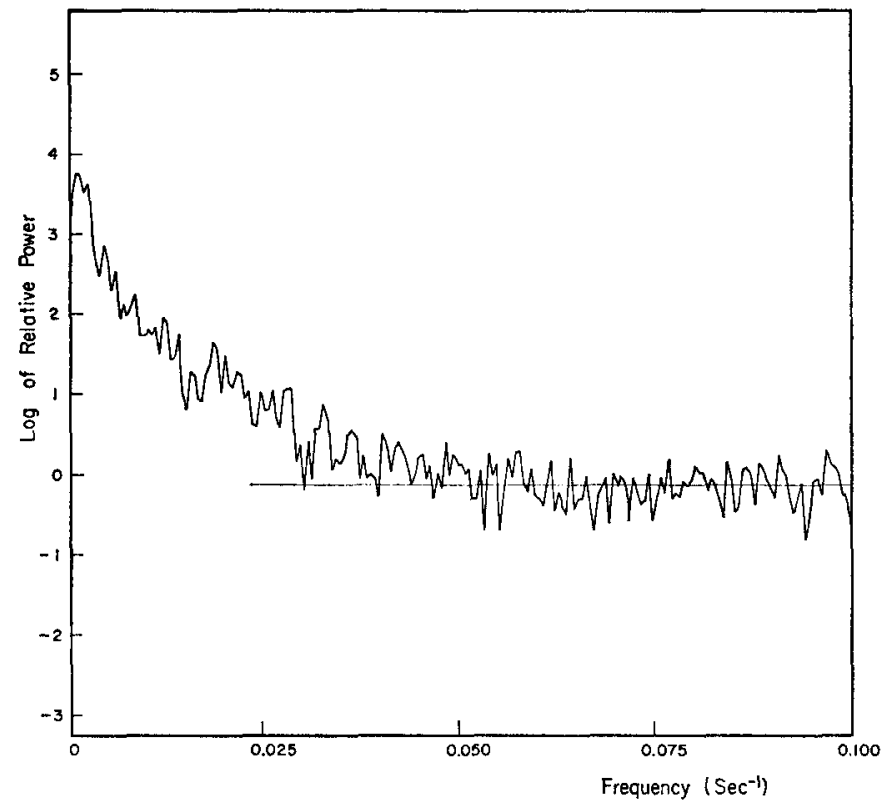

Fig. 12: Power spectrum of Sco XR-1. 
made by NATHER and WARNER (1969) used on-line signal averaging, storing ten complete periods with 20 channels per period. The instrumental cycle time was set at the geocentric period of DQ Her.

Some results are shown in Figure 13. Curves $a, b$ and $c$ are three typical summed light curves. It can be seen that even the averaging of ten complete periods still leaves considerable noise. Curve $d$ is the average of 22 of the summed light curves. Now the noise level has been reduced to the point where it is found that the light curve of the 71 second oscillation is very close to a sine wave (the continuous line in Figure 13).

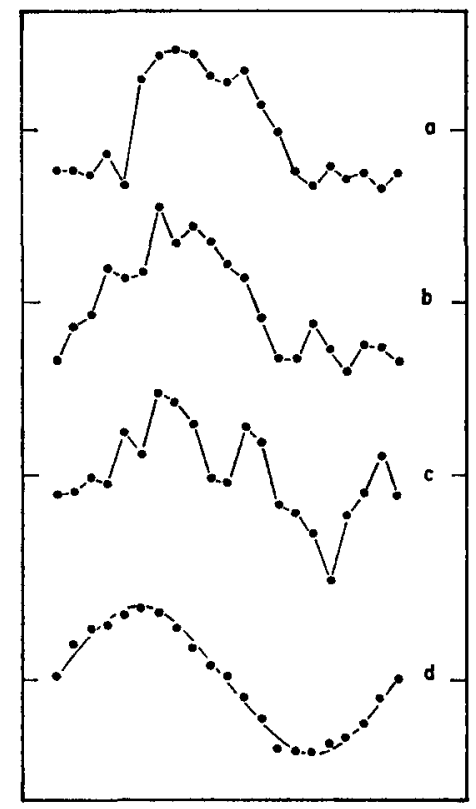

Fig. 13: Light curves of 71-second pulsations in DQ Her. See text for details.

In more recent (unpublished) work we have confirmed the general variation in amplitude of the 71 second light curve, discovered by WALKER (1961), around the binary orbit of DQ Her. Average light curves, as a function of position in orbit, indicate that the $71 \mathrm{sec}$ period light curve stays the same shape, but changes in amplitude.

Figure 14 illustrates the emergence from eclipse of the 71 second oscillations; five light curves are given. These data lead to a different conclusion than that reached in the previous work - namely, the oscillations reappear gradually over several cycles, not in less than one cycle as concluded by NATHER and WARNER (1969). The diameter of the white dwarf deduced from the eclipse phenomena is no longer in serious conflict with theoretical estimates.

\section{7) Synchronous Photometry of NP 0532}

Several hundred contiguous 1 minute integrations have been obtained of the pulsar in the Crab Nebula. The light curves were taken at $20 \mu \mathrm{sec}$ time resolution, the instrumental period being updated by the NOVA computer in 100 picosecond steps. The ephemeris prediction tape was generated by a separate program on the IBM 1800 at McDonald Observatory. Preliminary indications are that the slip in phase between instrumental and apparent pulsar period was about $0.5 \mu \mathrm{sec}(1 / 40$ of a channel) during a 1 minute integration. 


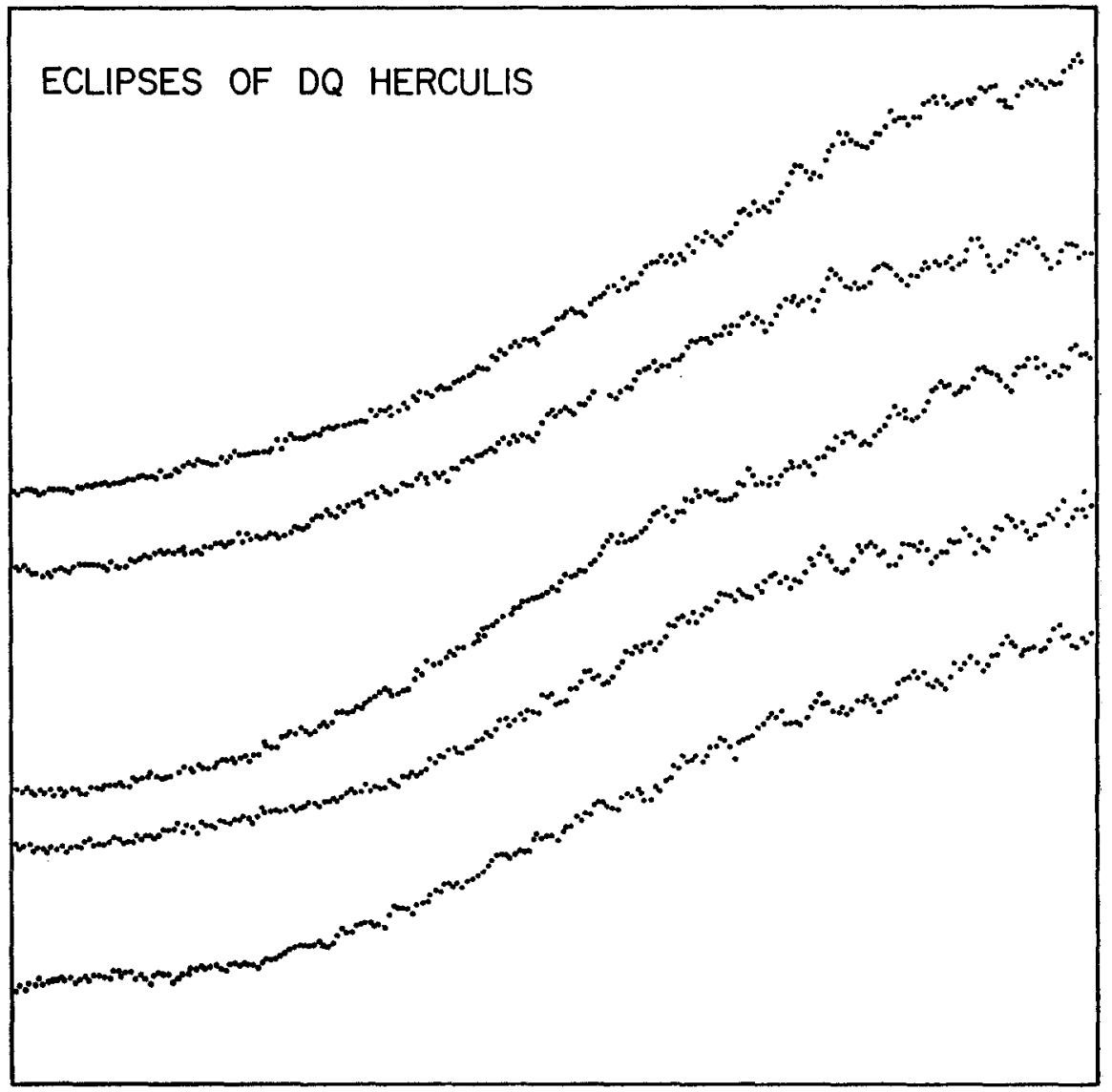

Each point represents $7 \mathrm{sec}$. Integration

Fig. 14: Eclipses of DQ Her.

A coaddition of approximately 1 hour of data appears in Figure 15. The ordinate scale is total detected photons. No attempt has been made here to adjust separate 1 minute integrations for phase shift. While nothing definite can be said about the high point at the peak of the pulse, data in hand have many suggestively high points at the peaks of separate 1 minute integrations. Reduced data may reveal some peak structure as well as information on jitter in the slowdown rate.

\section{Adknowledgements}

The work at McDonald Observatory described in this review has been a collaborative effort on the part of R. E. NATHER, G. W. VAN CITTERS, E. L. ROBINSON and the writer. This work has been partially supported by a research grant from the National Science Foundation. 


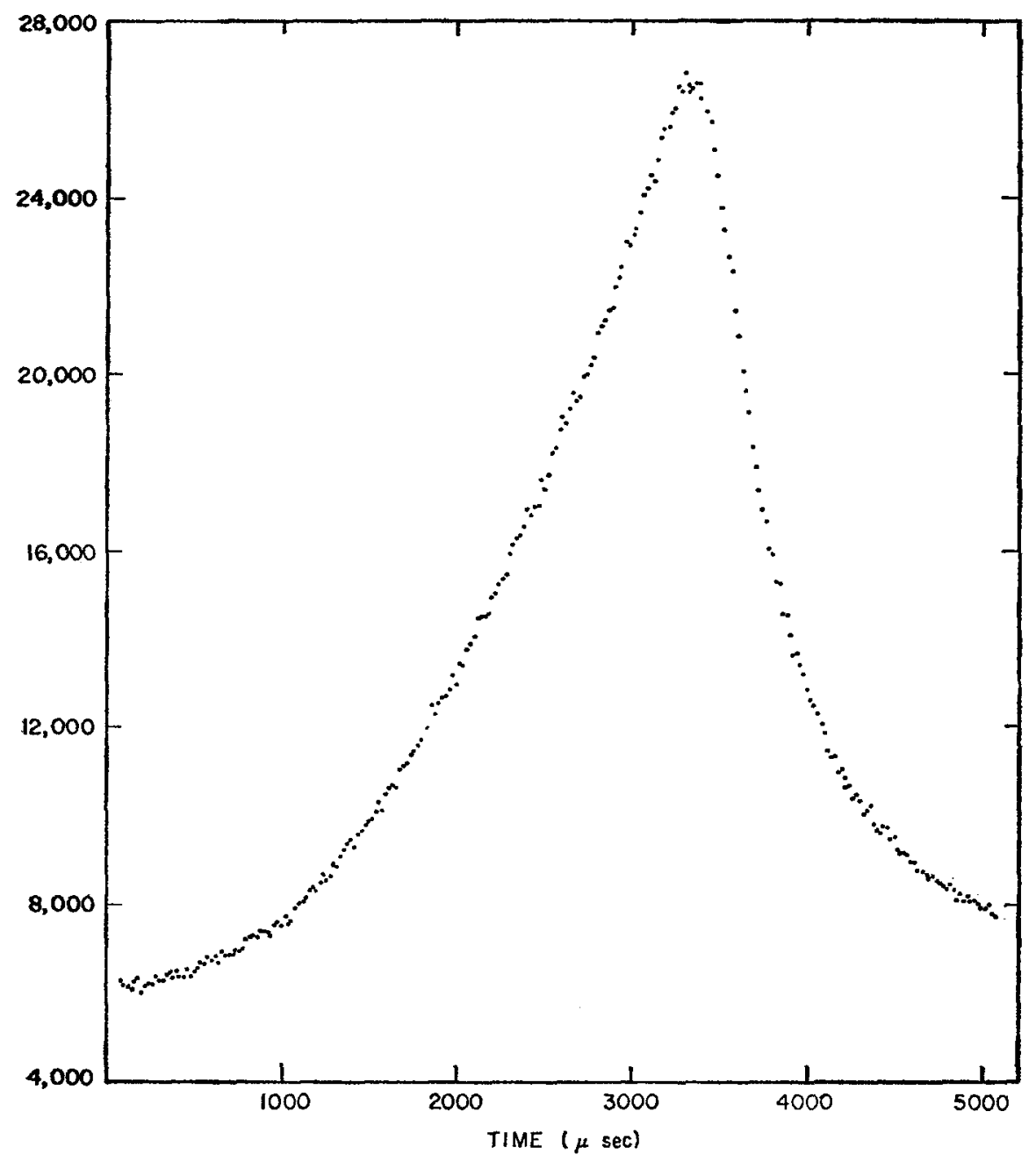

Fig. 15: Main pulse of NP 0532 at 20 microsecond time resolution. See text for details.

$$
\text { Ref erences: }
$$

COCKE, W. J., DISNEY, M. J., and TAYLOR, D. J., 1968, Nature 221, 525.

GREENSTEIN, J. L., 1965, Pub. Astr. Soc. Pacific 66, 71.

KRAFT, R. P., KRZEMINSKI, W., and MUMFORD, G. S., 1969, Astrophys. J. 154, 139.

KRZEMINSK I, W., 1965, Astrophys. J. 142, 501.

LAMPTON, M., BÖWYER, C. S., and HARRINGTON, S., 1970, Astrophys. J. 162, 181.

LANDOLT, A. U., 1968, Astrophys. J. 153, 151.

MUMFORD, G. S., 1963, Sky and Tel. 26, 190.

NATHER, R. E., and WARNER, B., 1969, Mon. Not. R. astr. Soc. 143, 145.

NATHER, R. E., and WARNER, B., 1971, Mon. Not. R. astr. Soc. 152, 209.

NATHER, R. E., WARNER, B., and MacFARLANE, M., 1969, Nature 221, 527.

PACZYNSKI, B., 1965. Acta Astron. 15, 305.

SERKOWSKI, K., 1970, Pub. Astr. Soc. Pacific 82, 908.

SMAK, J., 1971, Acta Ástron. 21, 15.

WALKER, M. F., 1954, Pub. Astr. Soc. Pacific 66, 71.

WALKER, M. F., 1956, Astrophys. J. 123, 68.

WALKER, M. F., 1957, I.A.U. Symposium No. 3, „Non-Stable Stars“, p. 46. 
WALKER, M. F., 1961, Astrophys. J. 134, 171.

WALKER, M. F., 1966, Adv, in Electronics. 228, 761.

WALKER, M. F., and CHINCARINI, G., 1968, Astrophys. J. 154, 157.

WARNER, B., and NATHER, R. E., 1970, Mon. Not. R. astr. Soc. 147, 21.

WARNER, B., and NATHER, R. E., 1971, Mon. Not. R. astr. Soc. 152, 219.

WESTPHAL, J. A., SANDAGE, A., and KRISTIAN, J., 1968, Astrophys. J. 154, 139.

\section{Discusston to the paper of WARNER}

HINDERER: The discoverer of U Gem, HINDERER, has observed at one of his first observations of the star a short-periodical flickering with an amplitude of ca. $1 \mathrm{~m}$. One has formerly believed, that this was an effect of anomaly of the atmosphere. Do you mean, that the observed flickering could possible be caused by the hot spot?

WARNER: The maximum amplitude of flickering that I have observed is $\sim 0.3$ magnitude. While 1 magnitude is out of the question, currently U Gem does not appear to be that active.

KELLOGG: How does the evidence for the binary nature of these variables described by you correlate with spectroscopic evidence?

WARNER: Many of the cataclysmic variables were first found to be double from spectroscopic evidence. There is perfect correlation between spectroscopic and photometric periods.

ARDEBERG: I would be interested to hear more about your photomultipliers and your ability to move one of them around freely.

WARNER: We use an Amperex 56 DVP in channel 1, and an RCA 4516 (miniature) photomultiplier on the second (moveable) dhannel.

ARDEBERG: So I understand that you do not use cooling. What is your typical differential sensitivity drift?

WARNER: We do not worry about slow timescale differential effects.

GEYER: The first observer who used the rapid comparison photometry was WALRAVEN in 1954, when he observed SX Phoe.

KUNKEL: Is the power law in the power spectrum of Sco X-1 similar in shape to that found by LASKER and HESSER for minimum light, that is, $\mathrm{n}=2$ in $\mathrm{P}(v) \propto v^{-\mathrm{n}}$ ?

WARNER: A first look at the data suggests that the answer is yes.

KUNKEL: What cross-correlation exists between the two non-varying stars?

WARNER: He have not yet looked at this.

MILEY: Have you observed any other X-ray sources besides Sco X-1?

WARNER: Yes, I have observed Cyg XR-2, but it was not active during my observations.

MUMFORD: Do you suspect or have you detected any other short periodicities similar to that shown for DQ Her?

WARNER: I have so far only suspected a $\sim 1$ min oscillation in UX UMa, but I have not yet carried out a periodogram analysis of the data.

HUTCHINGS: Do you have any comment on the 3 minute period found by power spectrum analysis of Sco XR-1, reported recently in Nature.

WARNER: Unless the same period is seen more than once, I feel that isolated examples of suspected periodicities may not have any physical significance.

BEHR: In the case of synchronized photometry where do you get the exact period from? How do you correct for apparent variations as a consequence of changing light time? How do you detect real changes in the period?

WARNER: In the case of the Crab Pulsar, the period was of course known in advance from the radio observations. The computer is programmed to update the pulsar-period in units of 100 picoseconds whenever required. If the pulsar period (or rate of slowing) is not regular this shows up in phase slippage with respect to our instrumental period. 